\title{
Patient and Physician Perspectives of Depressive Symptoms and Expectations for Treatment Outcome: Results from a Web-Based Survey
}

\author{
Jun Ishigooka id \\ Tatsuya Hoshino iD ${ }^{2}$ \\ Takumi Imai iD ${ }^{3}$ \\ Hisako Yoshida (iD ${ }^{3}$ \\ Midori Ono (iD ${ }^{2}$ \\ Mihoko Ota (iD) ${ }^{2}$ \\ Yoshiya Moriguchi (iD) ${ }^{4}$ \\ Keita Fujikawa (iD ${ }^{2}$ \\ Ayumi Shintani ${ }^{3}$ \\ Jovelle L Fernandez iD $^{2}$ \\ 'Institute of CNS Pharmacology, Tokyo, \\ Japan; ${ }^{2}$ Japan Medical Office, Takeda \\ Pharmaceutical Company Limited, Tokyo, \\ Japan; ${ }^{3}$ Department of Medical Statistics, \\ Osaka City University Graduate School \\ of Medicine, Osaka, Japan; ${ }^{4}$ Medical \\ Affairs, Lundbeck Japan K.K., Tokyo, Japan
}

Correspondence: Tatsuya Hoshino Japan Medical Office, Takeda

Pharmaceutical Company Limited, I-I,

Nihonbashi-Honcho 2-chome, Chuo-ku,

Tokyo, 103-8668, Japan

Tel +8I 332782111

Fax +8I 332782925

Email tatsuya.hoshino@takeda.com
Purpose: A previous international study suggested that perceptions of depression symptoms, social function, and treatment expectations are different between patients/physicians. We aimed to examine whether such differences exist in Japan.

Methods: A web-based survey was conducted with patients who reported that they had been diagnosed with depression, and physicians who reported that they had treated patients with depression, in Japan. Questionnaires were designed to quantify patients' perceptions of symptoms, social function, and treatment expectations. Patients were categorized into three stages of disorder based on their reported current symptoms: severe symptomatic, mild symptomatic, and remission. Physicians were assigned up to three patients, were provided with patient information from the questionnaire completed by those patients, and finally the completed questionnaire forms for each patient. Agreement between the perceptions of the patients and physicians was examined for each stage.

Results: Of the 2618 eligible patients, 828 were assigned to 326 eligible physicians. Overall, we found small differences in the perceptions of depression treatment between patients/ physicians. Slightly fewer physicians than patients reported physical symptoms $(85 \%$ vs $91 \% ; \mathrm{p}=0.018$ ) in the mild symptomatic stage. Fewer physicians than patients reported cognitive symptoms in the severe $(82 \%$ vs $87 \%$; $=0.029)$ and mild $(54 \%$ vs $66 \%$; $\mathrm{p}=0.003$ ) symptomatic stages. Social function was deemed to be lower by physicians than by patients, across all stages of disorder $(\mathrm{p}<0.001)$. Regarding treatment expectations, more physicians than patients reported "return to a normal life" in the mild symptomatic $(51 \%$ vs $35 \%, \mathrm{p}<0.001)$ and remission stages $(57 \%$ vs $36 \%, \mathrm{p}<0.001)$, and more patients than physicians reported "reduction of side effects" in the severe $(10 \%$ vs $4 \%, p=0.004)$ and mild $(12 \%$ vs $5 \%, \mathrm{p}<0.001)$ symptomatic disorder stages

Conclusion: These results suggest small differences in patient/physician perceptions of depression treatment in Japan. Discrepancies between patients'/physicians' perceptions may vary depending on the medical environment.

Keywords: depression, perception, patient, physician, web-based survey

\section{Introduction}

Depression is a mental health disorder that severely limits psychosocial functioning and diminishes patients' quality of life (QOL). ${ }^{1,2}$ Depression symptoms fall into a wide range of emotional, physical, and cognitive manifestations, which adversely affect the individual's life. ${ }^{2}$

Optimal management of depression requires the remission of mood symptoms as well as physical and cognitive symptoms. ${ }^{3}$ Clinical management of depression 
should focus on the patient's psychosocial functioning to improve social and family relationships and QOL during the remission phase. ${ }^{3,4}$

Psychotherapy and antidepressant therapy are wellestablished treatment options for patients with major depressive disorder (MDD), achieving remission in approximately 2 out of 3 patients. ${ }^{5}$ Most remitters, however, present with residual symptoms of depression, which strongly predicts subsequent relapse and MDD recurrence. ${ }^{6-10}$ Therefore, it is important that residual symptoms of depression are not overlooked. In this regard, differences between patient and physician perspectives of depression symptoms are an obstacle for sustained remission. ${ }^{11-14}$ Some patients who are in remission according to symptom severity scales still experience symptoms (at low levels), functional impairment, or deficits in coping ability. ${ }^{13}$ Patients with a poor patientphysician agreement, on treatment expectations, had worse clinical outcomes than those with an excellent patient-physician agreement. ${ }^{12}$ Therefore, there is an unmet need for a more formal, structured goal-setting process for MDD treatment plans and to facilitate better communication between patients and physicians. ${ }^{15}$ Understanding all of the treatment goals, for patients with depression, may lead to incorporation of clinically relevant outcome measures in treatment programs, thus improving treatments. ${ }^{14}$ These reports suggest the importance of both patients and physicians understanding each other's perceptions. ${ }^{11-14}$

Currently, there are limited data about the agreement/ disagreement between patients and physicians about symptoms, social functioning, and expectations of treatment. For example, discrepancies regarding severity, symptoms, and social functioning have been reported, with the proportion of patients with severe symptoms assessed as being higher by self-assessment of severity using the Center for Epidemiological Studies-Depression (CES-D) 20 -item scale, than by the physician's assessment. ${ }^{16}$ Furthermore, approximately $40 \%$ of patients who selfreported depression or anxiety were not identified as suffering from these symptoms, by their physicians. Additionally, patients perceived social function and QOL were lower when they were in disagreement with their physician diagnosing them as in remission..$^{13,17}$ In a further study, physicians and patients differed significantly in what they considered important in the treatment of depression: physicians primarily focused on alleviation of depressive symptoms while patients focused on restoration of positive affect. ${ }^{12}$ Although these findings are important, the limited number of studies and the variety of severity of depression and assessment methods indicate that further studies are needed.

Baune \& Christensen (2019) conducted a web-based survey to quantify potential differences between patients' and physicians' perceptions of depression across the three different disease stages, in eight countries, Brazil, Canada, Mexico, South Korea, United States, France, Italy, and Spain. ${ }^{11}$ Patients in the remission phase reported mood, physical, and cognitive symptoms more frequently than physicians. In the most recent analysis of this survey, patients and physicians were found to perceive the association between MDD symptoms and social functioning differently across each disease stage. ${ }^{18}$ These data substantiate the need for improved communication, between patients and physicians, to assess depressive symptoms and their impacts on patients across the different disorder stages to promote symptomatic and functional recovery in patients with depression. ${ }^{11,18}$ From the previous study, if the results universally applied to the treatment of depression in the psychiatric field, this would indicate a major problem in the treatment practice of depression. Therefore, it is important to confirm whether these results are reproducible, even in different regions and medical environments.

In this study, we conducted a web-based survey to assess the perceptions of symptoms, social functioning, and expectations for depression treatment across different stages of symptomatic relief (severe symptomatic, mild symptomatic, and remission), among patients with depression and physicians who treat depression, in Japan, with reference to the concept and methods of the previous study by Baune and Christensen (2019). ${ }^{11}$

\section{Materials and Methods Study Design}

The survey was carried out via closed web-based questionnaires, in Japan. The patient survey was conducted between March 30, 2020 and April 12, 2020. Survey participants were recruited from an existing online patient panel (https://insight.rakuten.co.jp/member/), owned and managed by Rakuten Insight Inc (Tokyo, Japan). The physician survey was conducted between April 17, 2020 and May 14, 2020. Psychiatrists and psychosomaticians were recruited from a physician panel (https://www.medi cal-ci.co.jp/en/research panel/), owned and managed by 
Medical Collective Intelligence Co., Ltd. (MCI Co., Ltd, Tokyo, Japan). Participants who responded to the questionnaires were remunerated by the company managing their panel.

\section{Ethics}

This study was conducted in accordance with the ethical principles of the Declaration of Helsinki and the International Conference on Harmonisation Guideline for Good Clinical Practice. The study protocol was approved by the ethical review committees of the Research Institute of Healthcare Data Science (Tokyo, Japan) before study initiation. All respondents consented to participate in the surveys and could discontinue the survey at any time. The study was registered on the University Hospital Medical Information Network Clinical Trials Registry (UMINCTR) before enrolling the first patient in the study (UMIN study ID: UMIN000039967).

\section{Inclusion and Exclusion Criteria}

Patients and physicians were screened for eligibility at the beginning of the survey. Patients who reported that they had been diagnosed with depression and had used antidepressants, in the past 3 months, were included. Eligible patients were aged $>19$ years and $<66$ years and had experienced a severe symptom of depression, as defined by a Japanese Patient Health Questionnaire (J-PHQ)-9 ${ }^{19}$ score of $\geq 10$, in the past (Supplemental Figure 1). Patients who were employed by a pharmaceutical company or marketing agency were excluded, as were patients reporting a J-PHQ-9 score of $<10$ as their most severe past depressive symptoms (Supplemental Figure 1).

Eligible physicians were board-certified psychiatrists or psychosomaticians, who reported that they examine $\geq 40$ patients with depression per month and prescribe antidepressants to $\geq 75 \%$ of these patients. Physicians working for a pharmaceutical company or marketing agency were excluded from the study.

\section{Assessments}

The physician and patient questionnaires were based on an English questionnaire used in a previous study, ${ }^{11}$ which was translated into Japanese for this study by ASCA Corporation (Osaka, Japan). After adjusting the wording to suit the clinical practice in Japan, the questionnaires were reviewed by at least five Japanese physicians and non-healthcare workers for the validity of the content. The patient survey comprised 12 screening and 26 survey questions, and assessed current symptoms of depression, symptoms that had not adequately improved, and expectations for treatment. The survey questions used are shown in Supplemental Table 1.

To assess the patient's current disease state, Baune and Christensen (2019) asked patients to carefully review three statements describing different phases of depression (acute, post-acute, and remission) and indicate which of these best described their current disease state. ${ }^{11}$ In this study, we classified the disorder stages as severe symptomatic, mild symptomatic, and remission. Severity of depressive symptoms was assessed using J-PHQ-9, ${ }^{19}$ a 9-item depression module extracted from the full $\mathrm{PHQ}^{20}$ and comprising the nine criteria upon which the diagnosis of the depressive disorders is based, according to the Diagnostic and Statistical Manual of Mental Disorders, 4th Edition. ${ }^{21}$ The patients were classified into three disorder stages based on their current J-PHQ-9 score, as follows: remission (1 or 0 ); mild symptomatic (2 to 9); and severe symptomatic (10 or higher) (Supplemental Figure 1). The cut-off-points were different from those recommended in the PHQ-9 manual, ${ }^{19,22}$ as we aimed to investigate the near-remission stage of the disorder.

Functioning problems were assessed using a modified version of the Functioning Assessment Short Test (FAST; with permission from the copyright owner, Professor Eduard Vieta, University of Barcelona, Spain), a 24-item questionnaire developed for the clinical evaluation of the main difficulties in daily functioning presented by psychiatric patients, ${ }^{23,24}$ which we adapted to suit an online survey. In the original FAST questionnaire, each respondent was asked to select the level of difficulty ("no difficulty," "mild difficulty," "moderate difficulty," "severe difficulty," or "don't know") associated with each of the 24 items relating to psychosocial functioning. In this study, we used the Japanese version of FAST translated by ASCA as described above.

The method of respondent allocation for the survey was modified from that used in Baune and Christensen (2019), ${ }^{11}$ in which physicians completed three patient record forms corresponding to the last patient treated for each of the three phases of depression being evaluated and were asked to refer to patient records when completing the patient record forms. In our study, a patient within each disorder stage was randomly assigned to a physician without replacement. Participating physicians were provided the following information for up to three patients in the patient cohort: age group, sex, period of current episode of depression, and answers to the J-PHQ-9 (assessing the current and most severe past depressive symptoms). The three patients 


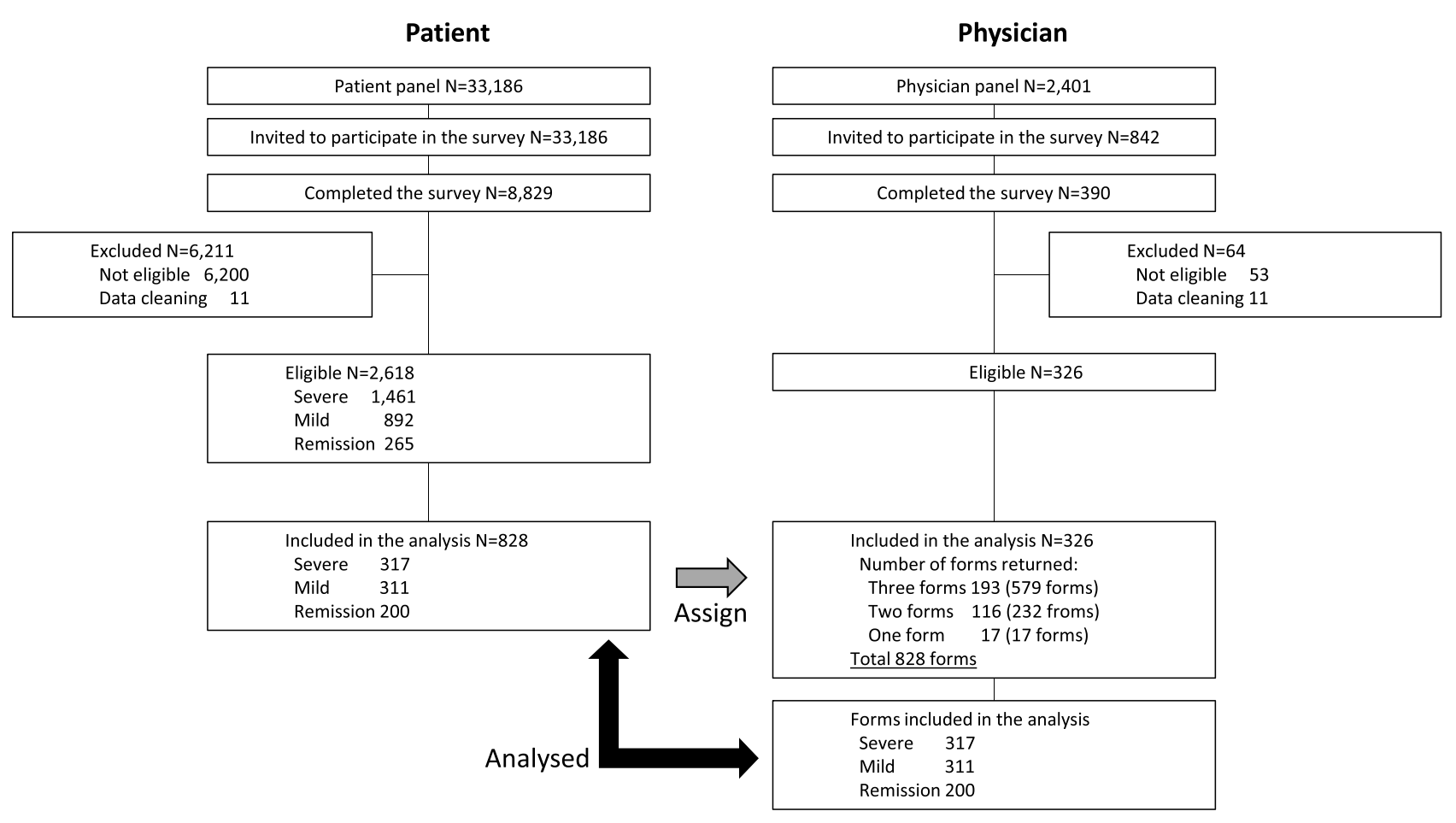

Figure I Survey flow diagram.

included one severe symptomatic patient, one mild symptomatic patient, and one patient in remission. The physicians were then asked to recall actual patients with similar attributes in his/her clinical practice, and the physicians would then refer to that recalled patient when answering a physician questionnaire, which included similar items to the patient questionnaire. The physicians' survey consisted of 6 screening and 15 survey questions per patient. Given that the physician was matched with up to three patients, the physician provided up to three responses, one for each correspondingly matched patient.

MCI Co., Ltd. was responsible for the collection, cleaning, and compilation of response data. Valid responses were defined as completed responses not meeting any exclusion criteria, and that did not have extremely short response times (ie, patient responses of $<5$ minutes, or physician responses of $<3$ minutes for one patient, $<6$ minutes for two patients, or $<9$ minutes for three patients) according to the standards of MCI Co., Ltd. All data obtained, including the information provided to physicians, were anonymized. Access to the data servers was limited to data administrators of MCI Co., Ltd.

\section{Statistical Analysis}

For the descriptive statistics, categorical variables were presented as frequencies and proportions; continuous variables were presented as means and standard deviation.
Differences in perceptions of the treatment of depression during the three phases of depression between patients and physicians were analyzed using generalized estimating equations that handled correlation among answers from matched patient-physician pairs and answers from the same physician. Covariates in the model included indicator variables for type of responder (patient or physician) and disorder stage (severe, mild, remission), and a crossproduct term between the indicator variables. Missing values were not imputed; only questionnaire forms with complete responses were analyzed. In the FAST questionnaire, respondents who selected "don't know" for five or more items were removed from the analysis of the FAST total scores, but the rest of their answers were retained for analysis of FAST subscale scores. SAS $^{\circledR}$ version 9.4 (M6) was used for all statistical analyses.

\section{Results}

\section{Participant Characteristics}

A total of 2618 patients and 326 physicians were eligible. From these, 317 severely symptomatic patients, 311 mildly symptomatic patients, and 200 patients in remission were randomly selected and assigned to physicians (Figure 1; see Supplemental Figure 1 for classification of disorder stage). The three groups had similar demographic characteristics (Table 1). Most patient baseline characteristics 
Table I Characteristics of Included Patients

\begin{tabular}{|c|c|c|c|}
\hline & $\begin{array}{l}\text { Severe } \\
(n=3 \mid 7)\end{array}$ & $\begin{array}{c}\text { Mild } \\
(n=3 I I)\end{array}$ & $\begin{array}{c}\text { Remission } \\
(n=200)\end{array}$ \\
\hline \multicolumn{4}{|l|}{ Age, n (\%) } \\
\hline $20-30$ years & $10(3.2)$ & $5(1.6)$ & $3(1.5)$ \\
\hline $31-50$ years & $181(57.1)$ & $138(44.4)$ & $79(39.5)$ \\
\hline $5 I-65$ years & $126(39.8)$ & $168(54.0)$ & $118(59.0)$ \\
\hline \multicolumn{4}{|l|}{ Sex, n (\%) } \\
\hline Male & $232(73.2)$ & 248 (79.7) & 175 (87.5) \\
\hline Female & $85(26.8)$ & $63(20.3)$ & $25(12.5)$ \\
\hline \multicolumn{4}{|l|}{ Educational qualification, n (\%) } \\
\hline University/college & $|3|(4 \mid .3)$ & $166(53.4)$ & $117(58.5)$ \\
\hline High school & $102(32.2)$ & $61(19.6)$ & $45(22.5)$ \\
\hline Junior/vocational college & $46(14.5)$ & $38(12.2)$ & $15(7.5)$ \\
\hline Postgraduate & $19(6.0)$ & $32(10.3)$ & $20(10.0)$ \\
\hline Junior high school & $10(3.2)$ & $5(1.6)$ & 0 \\
\hline Others & $9(2.8)$ & $9(2.9)$ & $3(1.5)$ \\
\hline \multicolumn{4}{|l|}{ Current job type, n (\%) } \\
\hline Full-time & $144(45.4)$ & $186(59.8)$ & $132(66)$ \\
\hline Unemployed & $81(25.6)$ & $42(13.5)$ & $17(8.5)$ \\
\hline Part-time & $49(15.5)$ & $29(9.3)$ & $19(9.5)$ \\
\hline Retired & $23(7.3)$ & $33(10.6)$ & $20(10)$ \\
\hline Stay-at-home & $20(6.3)$ & $21(6.8)$ & II (5.5) \\
\hline Student & 0 & 0 & $\mathrm{I}(0.5)$ \\
\hline Current PHQ-9 score, mean \pm SD & $16.9 \pm 5.3$ & $5.9 \pm 2.3$ & $0.5 \pm 0.5$ \\
\hline $\begin{array}{l}\text { Past worst PHQ-9 score, } \\
\text { mean } \pm \text { SD }\end{array}$ & $21.0 \pm 5.2$ & $19.8 \pm 5.2$ & $20.2 \pm 4.9$ \\
\hline $\begin{array}{l}\text { Time from first diagnosis (year), } \\
\text { mean } \pm \text { SD }\end{array}$ & $10.8 \pm 6.5$ & $9.5 \pm 6.6$ & $10.7 \pm 6.3$ \\
\hline
\end{tabular}

Abbreviations: N, number; PHQ-9, Patient Health Questionnaire-9; SD, standard deviation.

included in this survey are comparable to those from other surveys on depression. ${ }^{25}$ Typically, females are more likely than males to report that they have been diagnosed with depression, as supported by the previous study which was comprised of $61 \%$ females and $39 \%$ males. ${ }^{11}$ However, most participants in this study were male (males, $\mathrm{n}=655$ [79\%]: females, $\mathrm{n}=173,[21 \%]$ ). Although the definite reason is not clear, the discrepancy may be due to a gender differentiated willingness to participate in web surveys. Among the physicians, $96 \%$ were psychiatrists and $4 \%$ were psychosomaticians, all of whom were psychiatric specialists. The majority of the physicians worked in a hospital setting (Table 2).

\section{Perception of Symptoms}

There were small differences in the perceptions of mood symptoms between patients and physicians at all disorder
Table 2 Characteristics of Included Physicians

\begin{tabular}{|l|c|}
\hline & $\begin{array}{c}\text { Physicians } \\
\text { (N=326) }\end{array}$ \\
\hline $\begin{array}{l}\text { Number of patients the physician sees per month, } \\
\text { mean } \pm \text { SD }\end{array}$ & $101 \pm 89$ \\
\hline $\begin{array}{l}\text { Percentage of patients the physicians prescribe } \\
\text { antidepressants to, mean } \pm \text { SD }\end{array}$ & $90.4 \pm 7.5$ \\
\hline $\begin{array}{l}\text { Specialty, n (\%) } \\
\text { Psychiatry }\end{array}$ & $313(96.0)$ \\
Psychosomatic medicine & $13(4.0)$ \\
\hline $\begin{array}{l}\text { Institution type, n (\%) } \\
\text { Hospital } \\
\text { Clinic and hospital }\end{array}$ & $187(57.4)$ \\
Clinic & $79(24.2)$ \\
\hline
\end{tabular}

Abbreviations: N, number; SD, standard deviation.

stages (Figure 2). However, physicians reported fewer physical symptoms than patients with mild symptoms ( $85 \%$ vs $91 \%$; $\mathrm{p}=0.018$ ), and fewer cognitive symptoms than patients with severe symptoms ( $82 \%$ vs $87 \%$; $\mathrm{p}=0.029)$ and those with mild symptoms (54\% vs $66 \%$; $\mathrm{p}=0.003$ ) (Figure 2). Patients in remission reported fewer cognitive symptoms compared with patient rates reported by physicians; however, the difference did not reach statistical significance (Figure 2). Examination of domains of cognitive symptoms showed that patients in some disorder stages reported higher rates of difficulty concentrating and forgetfulness/difficulty remembering, than patient rates reported by physicians (Supplemental Figure 2).

Compared with patients, physicians reported higher rates of lack of improvement in patients' mood symptoms in the mild and remission stages, and higher rates of lack of improvement in cognitive symptoms in the mild stage (Supplemental Table 2). Physicians reported mood as a symptom that patients wanted to treat at a higher rate than patients with mild symptoms (Supplemental Table 3).

\section{Social Functioning}

For all disorder stages, physicians rated mean FAST total scores (reflecting low social functioning) higher than patients (severe symptomatic stage, 49 vs $40, \mathrm{p}<0.001$; mild symptomatic stage, 30 vs $19, p<0.001$; remission, 13 vs $6, p<0.001$ ) (Figure 3). All individual subdomains of FAST were also rated low on social functioning by physicians, for patients at all disorder stages (Supplemental Figure 3). 


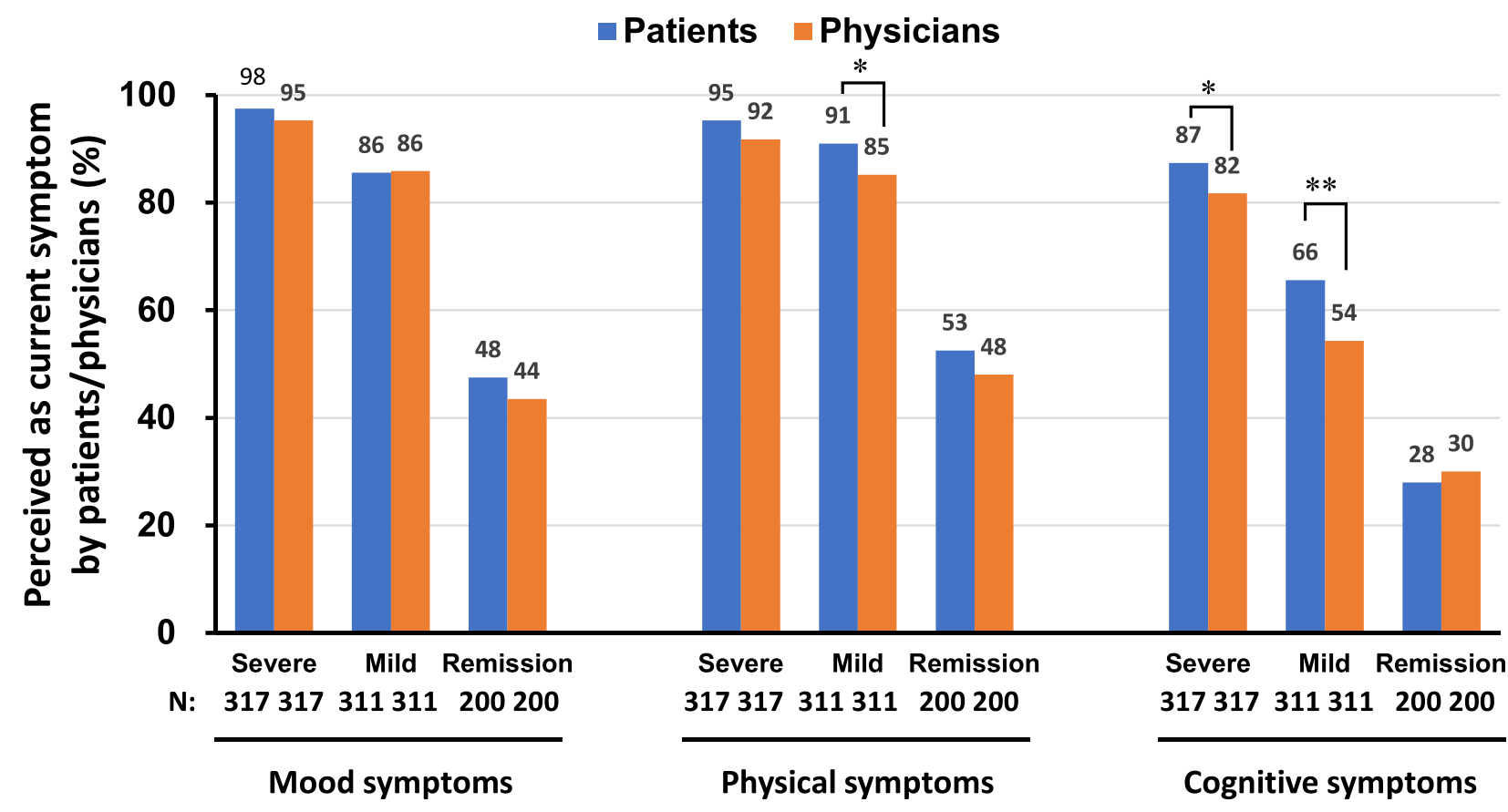

Figure 2 Perceptions of current symptoms at each disorder stage between patients and physicians. Notes: ${ }^{*} p<0.05,{ }^{*} p<0.01$.

Abbreviation: $\mathrm{N}$, number.

\section{Expectations for Treatment}

The proportion of respondents who expected treatment to provide a "return to a normal life" increased from the severe symptomatic stage to the remission stage, and more so with physicians than with patients (severe symptomatic stage, $31 \%$ for patients and $31 \%$ for physicians, $\mathrm{p}=0.930$; remission stage, $36 \%$ for patients and $57 \%$ for physicians, $\mathrm{p}<0.001)$. In contrast, more patients expected a "reduction of side effects" in the severe and mild stages (severe symptomatic stage, $10 \%$ for patients and $4 \%$ for physicians, $\mathrm{p}=0.004$; mild symptomatic stage, $12 \%$ for patients and $5 \%$ for physicians, $\mathrm{p}<0.001$ ) (Figure 4).

\section{Discussion}

This survey showed that there were small differences in the perception of depression symptoms, symptoms not being adequately improved, and symptoms that patients wanted treatment for, between patients and physicians in Japan. These results were different from those of the previous study conducted by Baune and Christensen (2019), ${ }^{11}$ which showed that patients in remission reported mood, physical, and cognitive symptoms more frequently than physicians. In our survey, patients also reported more symptoms not being adequately improved and more symptoms that they wanted treated in all disorder stages. Furthermore, in this current study, physicians consistently rated social functioning lower than the patients did across all stages. These findings on social functioning were the opposite of those in the previous study, ${ }^{11}$ which showed that patients reported a greater impact of symptoms on social functioning across all disorder stages, than physicians. Although there was some lack of agreement between physicians and patients when comparing the detail of each symptom and expectation for treatment outcomes, there was no consistent trend in stage-dependency in this study.

All the physicians who participated in this study were specialists in psychiatry whereas about one-third of physicians who participated in the previous study were not; ${ }^{11}$ this was due to differences in the respective inclusion criteria. This discrepancy in the proportion of specialists may have contributed to differences between the results of this and the previous study. Different evaluations between patients and physicians can be potential obstacles to the treatment of depression and could be resolved by improving the quality of medical care and the medical system.

The difference in perception of social function was more consistently observed in all disorder stages in 


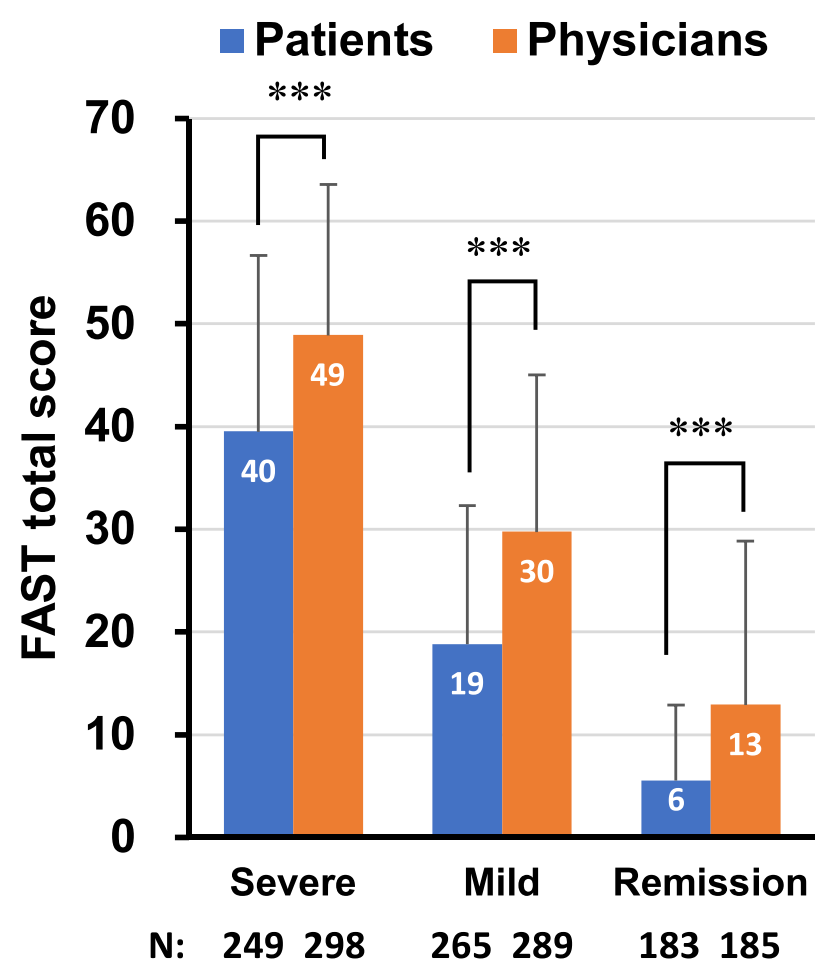

Figure 3 Social functioning assessed by FAST at each disorder stage between patients and physicians. Values represent mean + standard deviation. In the FAST questionnaire, respondents who selected "don't know" for five or more items were removed from the analysis of the FAST total scores, but the rest of their answers were retained for analysis of FAST subscale scores (Supplemental Figure 3). Note: $* * * p<0.001$.

Abbreviations: N, number; FAST, Functioning Assessment Short Test.

Japan. Physicians have noted that recovery of function is not as easy to achieve as patients expect. ${ }^{26,27}$ There is a well-known lag between the improvement of mood symptoms and functional recovery, ${ }^{27}$ and standard cutoffs of symptom severity scales used to define remission may be too high. ${ }^{26,27}$ On the other hand, patients who are in, or approaching, the remission stage tend to be optimistic about their social functional recovery. Lack of awareness of functional impairment in the mild symptomatic to remission stages may lead the patient to prematurely return to work, thereby increasing the risk of worsening of mood symptoms or relapse of depression. It is therefore important to improve this perception gap in the evaluation of social functioning between the patients and the physicians during the treatment of depression to achieve therapeutic outcomes.

While there were small differences in the perceptions of patients and physicians regarding symptoms and expectations for treatment of depression overall, statistically significant differences existed for some disorder stages. These results indicate that symptom evaluation is not always consistent between patients and physicians, and thus, physicians should pay greater attention to the patient's self-evaluation in the process of treatment of depression.

Although this study provides valuable information on differences in perception between patients and physicians, some limitations need to be taken into consideration when comparing with the previous study by Baune \& Christensen (2019). ${ }^{11}$ First, questionnaires used in this study, including FAST, were simply translated into Japanese and the wording was modified to suit clinical practice in Japan. Thus, the reliability and validity of the questionnaire have not been sufficiently confirmed. Second, the criteria for patient disorder stages were modified to reflect our interest in this study. Briefly, in the previous study, patients subjectively chose their disorder stage, but in this study, they were classified according to the PHQ-9 score for quantitative staging. Third, although there was no pairing between each patient's and physician's answers in the previous study, in this study, patients' and physicians' answers were paired answers of patients and physicians in this study (through sharing of a part of the patient information with the physician) to allow pairwise comparisons.

This study also includes a number of clinical limitations: the exact medication history of the patients was not obtained, and some patients may have been taking more than one type of antidepressant, or not have been taking on them long enough to reveal the true impacts of the medication. This may have altered the patient's overall perceptions. Additionally, diet, exercise, and meditation can also help depressive symptoms. These aspects of the patients' lives were not considered in this study. Furthermore, it should be acknowledged that surveys, such as this, present a limited snapshot of a patient's mental state, and not a complete picture of the patient's experience or associated feelings over time. While this study has provided insights into differences in patient perceptions of their depression, a more in-depth analysis of patient attitudes over time would help to further support our findings. A further limitation of this study is that physician responses were based upon recollection of patient results that they perceived as similar to those in the patient records provided, but not that actual patient. Physicians consulting with patients in real life, would ask more questions considering factors such as family medical history and individual demographic factors. Future research should examine more indepth one-to-one encounters between patients and 


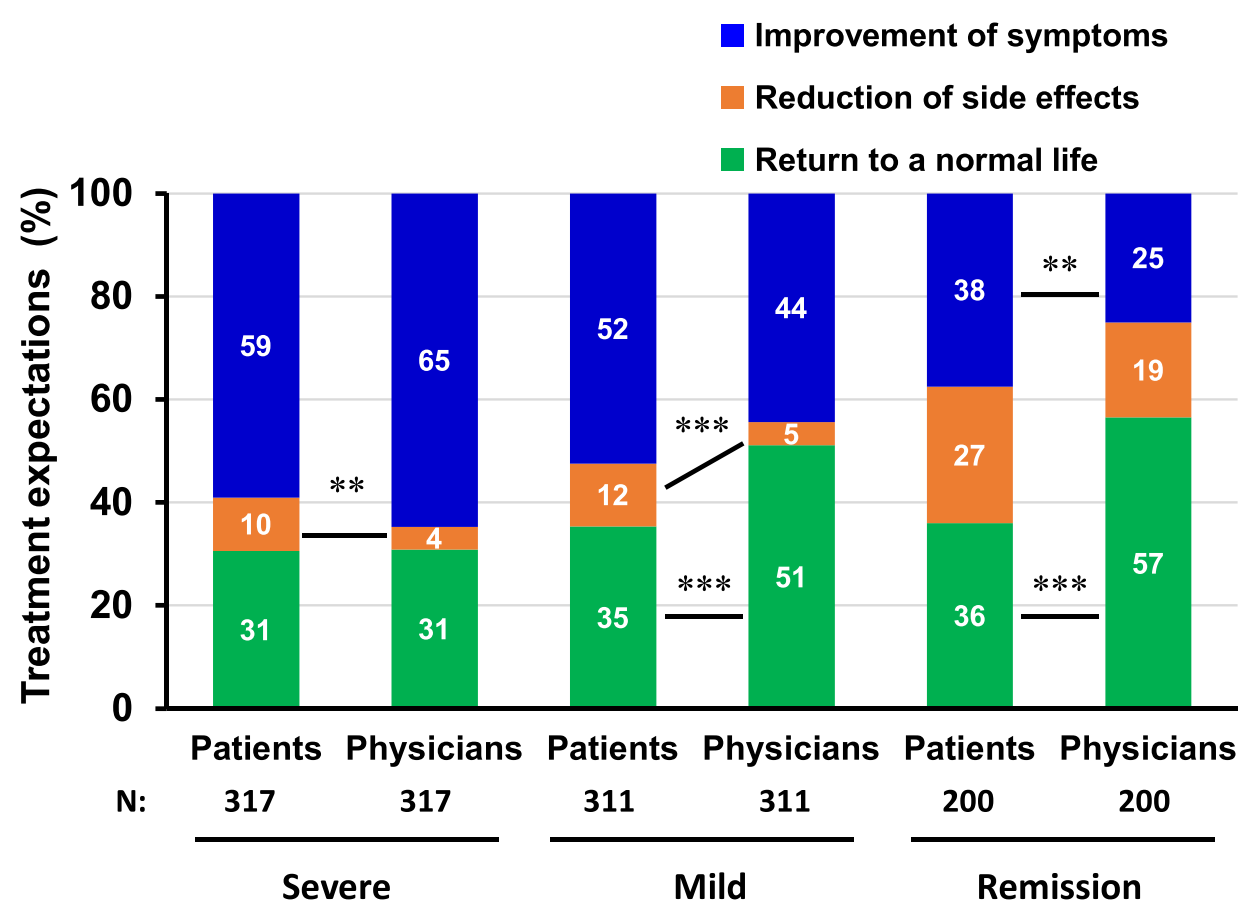

Figure 4 Patients' and physicians' expectations from the treatment of symptoms at each disorder stage.

Notes: ** $p<0.01$, *** $p<0.001$.

Abbreviation: $\mathrm{N}$, number.

physicians, to establish if any major perception differences emerge. Whilst with limitations, the authors believe that this study provides useful information regarding perceptions of patients and physicians, which may provide helpful insights towards management of depression which may be unique to Japan.

\section{Conclusions}

This study reports the current perceptions of patients and physicians regarding the symptoms and treatment of depression, in Japan. These perceptions seem to vary depending on the medical environment. Small differences in the perceptions between patients and physicians were revealed. At all disorder stages, physicians rated social functioning lower than patients, suggesting that patients who are in, or approaching, the remission stage may be overly optimistic about their social function. These results suggest that improving patient awareness of impaired social functioning may be warranted. Although there were some differences between patients' and physicians' perceptions when comparing each symptom and expectation for treatment outcome in detail, there was no consistent trend in stage-dependency. This study contributes to the understanding of current perceptions of patients and physicians regarding treatment of depression, in Japan.

\section{Acknowledgments}

The study was sponsored by Takeda Pharmaceutical Co. Ltd. (Osaka, Japan) and Lundbeck Japan K.K. (Tokyo, Japan). The authors acknowledge the contributions of Hiroshi Tsuda, Takafumi Akimoto, and Medical Collective Intelligence Co., Ltd. Medical writing support was provided by Ivan Olegario and Fumiko Shimizu of MIMS Pte Ltd., which was funded by Takeda Pharmaceutical Company Limited (Osaka, Japan) and Lundbeck Japan K.K. (Tokyo, Japan), and complied with Good Publication Practice 3 ethical guidelines (Battisti et al Ann Intern Med. 2015; 163: 461-4).

\section{Disclosure}

Dr. Ishigooka reports honoraria received for advisory boards/lectures/papers and/or research funding from Takeda Pharmaceutical, Lundbeck Japan, Otsuka Pharmaceutical, Eli Lilly Japan, Yoshitomi Pharmaceutical, Novartis Japan, Alfresa, Janssen Pharmaceutical, and Astellas Pharma. Dr. Hoshino, Ms Ono, Ms Ota, Dr. Fujikawa and Dr. Fernandez are employees of Takeda Pharmaceutical Company Limited. Dr. Fernandez holds restricted shares from GSK and Takeda Pharmaceutical Company Limited. Dr. Moriguchi is an employee of 
Lundbeck Japan KK. Prof. Shintani reports honoraria received for advisory boards/lectures/papers and/or research funding from Takeda Pharmaceutical Company Limited, Shionogi, Daiichi Sankyo, Bristol Myers Squibb, Kyowa Kirin, Chugai Pharmaceutical, Janssen Pharmaceutical, and Fujiyakuhin. The authors report no other conflicts of interest in this work.

\section{References}

1. Kennedy SH. Core symptoms of major depressive disorder: relevance to diagnosis and treatment. Dialogues Clin Neurosci. 2008;10 (3):271-277. doi:10.31887/DCNS.2008.10.3/shkennedy

2. Culpepper L, Muskin PR, Stahl SM. Major depressive disorder: understanding the significance of residual symptoms and balancing efficacy with tolerability. $A m \quad J$ Med. 2015;128(9):S1-S15. doi:10.1016/j.amjmed.2015.07.001

3. Greer TL, Kurian BT, Trivedi MH. Defining and measuring functional recovery from depression. CNS Drugs. 2010;24(4):267-284. doi:10.2165/11530230-000000000-00000

4. Saragoussi D, Christensen MC, Hammer-Helmich L, Rive B, Touya M, Haro JM. Long-term follow-up on health-related quality of life in major depressive disorder: a 2-year European Cohort Study. Neuropsychiatr Dis Treat. 2018;14:1339-1350. doi:10.2147/NDT. S159276

5. Rush AJ, Trivedi MH, Wisniewski SR, et al. Acute and longer-term outcomes in depressed outpatients requiring one or several treatment steps: a STAR*D report. Am J Psychiatry. 2006;163(11):1905-1917. doi:10.1176/ajp.2006.163.11.1905

6. Nierenberg AA, Husain MM, Trivedi MH, et al. Residual symptoms after remission of major depressive disorder with citalopram and risk of relapse: a STAR*D report. Psychol Med. 2010;40(1):41-50. doi:10.1017/S0033291709006011

7. Saragoussi D, Touya M, Haro JM, et al. Factors associated with failure to achieve remission and with relapse after remission in patients with major depressive disorder in the PERFORM Study. Neuropsychiatr Dis Treat. 2017;13:2151-2165. doi:10.2147/NDT. S136343

8. Verhoeven FEA, Wardenaar KJ, Ruhé HGE, Conradi HJ, de Jonge P. Seeing the signs: using the course of residual depressive symptomatology to predict patterns of relapse and recurrence of major depressive disorder. Depress Anxiety. 2017;35(2):148-159. doi:10.1002/ da. 22695

9. Albert U, Brugnoli R, Caraci F, et al. Italian psychiatrists' perception on cognitive symptoms in major depressive disorder. Int J Psychiatry Clin Pract. 2015;20(1):2-9. doi:10.3109/13651501.2015.1093147

10. McAllister-Williams RH, Bones K, Goodwin GM, et al. Analysing UK clinicians' understanding of cognitive symptoms in major depression: a survey of primary care physicians and psychiatrists. $J$ Affect Disord. 2017;207:346-352. doi:10.1016/j.jad.2016.09.036

11. Baune BT, Christensen MC. Differences in perceptions of major depressive disorder symptoms and treatment priorities between patients and health care providers across the acute, post-acute, and remission phases of depression. Front Psychiatry. 2019;10:335. doi:10.3389/fpsyt.2019.00335

12. Demyttenaere K, Donneau A-F, Albert A, Ansseau M, Constant E, van Heeringen $\mathrm{K}$. What is important in being cured from depression? Discordance between physicians and patients (1). J Affect Disord. 2015;174:390-396. doi:10.1016/j.jad.2014.12.004
13. Zimmerman M, Martinez JA, Attiullah N, et al. Why do some depressed outpatients who are in remission according to the Hamilton depression rating scale not consider themselves to be in remission? J Clin Psychiatry. 2012;73(6):790-795. doi:10.4088/ jcp. $11 \mathrm{~m} 07203$

14. Uebelacker LA, Battle CL, Friedman MA, Cardemil EV, Beevers CG, Miller IW. The importance of interpersonal treatment goals for depressed inpatients. J Nerv Ment Dis. 2008;196 (3):217-222. doi:10.1097/nmd.0b013e3181663520

15. McNaughton EC, Curran C, Granskie J, et al. Patient attitudes toward and goals for MDD treatment: a Survey Study. Patient Prefer Adherence. 2019;13:959. doi:10.2147/PPA.S204198

16. Marando F, Gualberti G, Costanzo A, et al. Discrepancies between physician's perception of depression in HIV patients and selfreported CES-D-20 assessment: the DHIVA Study. AIDS Care. 2016;28(2):147-159. doi:10.1080/09540121.2015.1080794

17. McGrady A, Lynch DJ, Nagel RW, Tamburrino M. Coherence between physician diagnosis and patient self reports of anxiety and depression in primary care. J Nerv Ment Dis. 2010;198(6):420-424. doi:10.1097/NMD.0b013e3181e084ce

18. Christensen MC, Wong CMJ, Baune BT. Symptoms of major depressive disorder and their impact on psychosocial functioning in the different phases of the disease: do the perspectives of patients and healthcare providers differ? Front Psychiatry. 2020;11:280. doi:10.3389/fpsyt.2020.00280

19. Muramatsu K, Miyaoka H, Kamijima K, et al. Performance of the Japanese version of the patient health questionnaire-9 (J-PHQ-9) for depression in primary care. Gen Hosp Psychiatry. 2018;52:64-69. doi:10.1016/j.genhosppsych.2018.03.007

20. Spitzer RL, Kroenke K, Williams JB. Validation and utility of a self-report version of PRIME-MD: the PHQ Primary Care Study. Primary care evaluation of mental disorders. patient health questionnaire. JAMA. 1999;282(18):1737-1744. doi:10.1001/ jama.282.18.1737

21. American Psychiatric Publishing, Inc. Diagnostic and Statistical Manual of Mental Disorders. 4th ed. American Psychiatric Publishing, Inc.; 1994:xxvii, 886-xxvii, 886.

22. Kroenke K, Spitzer RL, Williams JB. The PHQ-9: validity of a brief depression severity measure. J Gen Intern Med. 2001;16(9):606-613. doi:10.1046/j.1525-1497.2001.016009606.x

23. Baune BT, Air T. Clinical, functional, and biological correlates of cognitive dimensions in major depressive disorder - rationale, design, and characteristics of the cognitive function and mood study (CoFaM-Study). Front Psychiatry. 2016;7:150. doi:10.3389/ fpsyt.2016.00150

24. Knight MJ, Air T, Baune BT. The role of cognitive impairment in psychosocial functioning in remitted depression. J Affect Disord. 2018;235:129-134. doi:10.1016/j.jad.2018.04.051

25. Noguchi R, Sekizawa Y, So M, Yamaguchi S, Shimizu E. Effects of five-minute internet-based cognitive behavioral therapy and simplified emotion-focused mindfulness on depressive symptoms: a randomized controlled trial. BMC Psychiatry. 2017;17(1):85. doi:10.1186/s12888-017-1248-8

26. Romera I, Perez V, Menchon JM, Polavieja P, Gilaberte I. Optimal cutoff point of the Hamilton rating scale for depression according to normal levels of social and occupational functioning. Psychiatry Res. 2011;186(1):133-137. doi:10.1016/j.psychres.2010.06.023

27. Sheehan DV, Nakagome K, Asami Y, Pappadopulos EA, Boucher M. Restoring function in major depressive disorder: a systematic review. $J$ Affect Disord. 2017;215:299-313. doi:10.1016/j.jad.2017.02.029 


\section{Publish your work in this journal}

Neuropsychiatric Disease and Treatment is an international, peerreviewed journal of clinical therapeutics and pharmacology focusing on concise rapid reporting of clinical or pre-clinical studies on a range of neuropsychiatric and neurological disorders. This journal is indexed on PubMed Central, the 'PsycINFO' database and CAS, and is the official journal of The International Neuropsychiatric Association (INA). The manuscript management system is completely online and includes a very quick and fair peer-review system, which is all easy to use. Visit http://www.dovepress.com/testimonials.php to read real quotes from published authors.

Submit your manuscript here: https://www.dovepress.com/neuropsychiatric-disease-and-treatment-journal 